\title{
Gut microbiota influence stroke recovery in mice
}
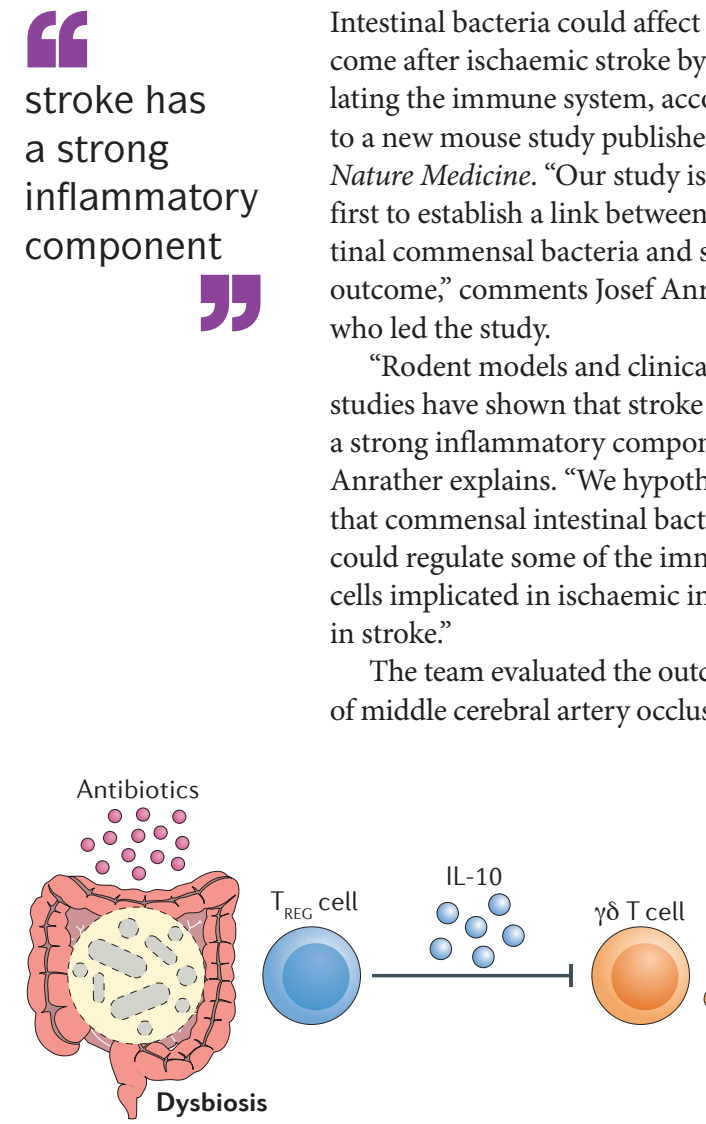

Intestinal bacteria could affect outcome after ischaemic stroke by regulating the immune system, according to a new mouse study published in Nature Medicine. "Our study is the first to establish a link between intestinal commensal bacteria and stroke outcome," comments Josef Anrather, who led the study.

"Rodent models and clinical studies have shown that stroke has a strong inflammatory component," that commensal intestinal bacteria could regulate some of the immune cells implicated in ischaemic injury in stroke."

The team evaluated the outcome of middle cerebral artery occlusion in Anrather explains. "We hypothesized mice in which dysbiosis was induced by antibiotics, and in control mice with antibiotic-resistant gut microbiota. The volume of the stroke infarct was $60 \%$ smaller in the dysbiotic mice than in the control mice; moreover, sensorimotor function was better preserved in the dysbiotic mice.

The investigators discovered that the effect of dysbiosis on stroke recovery was mediated by $\mathrm{T}$ cells. In eubiotic mice, stroke triggered migration of IL-17-secreting $\gamma \delta$ $\mathrm{T}$ cells from the intestinal lamina propria to the meninges, where they promoted inflammation and exacerbated injury. Antibiotic treatment increased the number of $\mathrm{T}_{\mathrm{REG}}$ cells in the lamina propria of the small
Dysbiosis increases the number of $\mathrm{T}_{\mathrm{REC}}$ cells, which secrete IL-10, thereby suppressing the development of intestinal IL-17-secreting $\gamma \delta$ T cells. A reduced IL-17 level inhibits poststroke inflammation and limits ischaemic brain injury. Image courtesy of C. Benakis. intestine; these cells secrete IL-10, which suppresses the development of $\mathrm{IL}-17^{+} \gamma \delta$ T cells, thereby reducing neuroinflammation.

Some previous human studies have reported poststroke increases in IL-17 levels, possibly secreted by infiltrated $\gamma \delta$ T cells. However, no epidemiological data has linked intestinal microbiota with the risk or outcome of stroke as yet.

"As more epidemiological data on the human microbiome become available, we can ask the question of whether a certain microbial makeup is correlated with the risk of cardiovascular events and - eventually - with stroke outcome," Anrather hopes. "Once such microbial traits are identified, it could be possible to develop a dietary or probiotic intervention to establish a flora with a beneficial effect on the cardiovascular system."

Hemi Malkki

ORIGINAL ARTICLE Benakis, C. et al. Commensal microbiota affects ischemic stroke outcome by regulating intestinal $\gamma \delta$ T cells. Nat. Med. http:// dx.doi.org/10.1038/nm.4068 (2016) FURTHER READING Fu, Y. et al. Immune interventions in stroke. Nat. Rev. Neurol. 11, 524-535 (2015) 